\title{
Controllability of Multiple Input Discrete-Time Linear Systems with Positive Controls ${ }^{\dagger}$
}

\author{
Hiroshi YoshIDA*, Yoshihiro TANADA*, \\ Tetsuro TANAKA* and Kazutomo YUNOKUCHI*
}

\begin{abstract}
The controllability with input constraints is important in practical problems. Especially, the controllability with positive controls is useful in discussing the vibration control problem of a pendulum system, economic system, etc. And so, this paper presents a necessary and sufficient condition for the controllability to the zero state by positive controls of multiple input discrete-time linear systems. It is derived that the positive controllability of a given system may be reduced to those of its subsystems corresponding to the the Jordan canonical form. Since the sizes of the subsystems are smaller than that of a given system, then the positive controllability test can be simplified very much. Furthermore, the structure of a positive controllable system is revealed. The results in this paper are fundamental and essensial in the control system theory.
\end{abstract}

Key Words : positive controls, controllability to the zero state, constrained controls, linear discrete-time systems, multiple input systems

\section{Introduction}

Many works concerning the controllability have been done since the concept of controllability was introduced by Kalman ${ }^{1), 2}$. The controllability with input constraints is important in dealing with practical problems. Especially, the controllability with positive controls is useful in discussing the control problem of a pendulum system, economic system, etc $^{3) \sim 5}$. Evance and Murthy presented the necessary and sufficient condition for the controllability of single input discrete-time linear systems with positive controls ${ }^{6)}$.

In general, there are two types of definitions of the controllability in regard to the desired state.

(1) Controllability to any state.

(2) Controllability to the zero state.

Evance and Murthy discussed the former case. On the other hand, the latter case is important in regard to a regulator problem. Yoshida studied the latter

$\dagger$ Presented 31th SICE Annual Conference (1992•7)

* Faculty of Engineering, Kagoshima University,

Korimoto 1-21-40, Kagoshima

(Received November 30, 1992)

(Revised September 20, 1993) case for single input systems ${ }^{7}$. The purpose of this paper is to give the necessary and sufficient conditions for the controllability to the zero state by positive controls of multiple input discrete-time linear systems.

\section{Preliminaries}

Consider a multiple input linear discrete time system described by

$$
S: \boldsymbol{x}(k+1)=\boldsymbol{A} \boldsymbol{x}(k)+\boldsymbol{B} \boldsymbol{u}(k) ; k=0,1,2, \cdots
$$

where

$$
\begin{aligned}
& \boldsymbol{A} \in R^{n \times n}, \quad \boldsymbol{B} \in R^{n \times m}, \\
& \boldsymbol{x}(k) \in R^{n}, \boldsymbol{u}(k) \in R^{m}
\end{aligned}
$$

The control input is limited to the following condition $C$.

$$
C: u_{i}(k) \geqq 0 ; i=1,2, \cdots, m
$$

where $u_{i}(k)$ is the $i$-th component of $\boldsymbol{u}(k)$.

Definition 1: The control input which satisfies (2) is called a positive control.

Definition 2: Let $x(0)$ be any initial state. Then system $S$ is called to be positive controllable, if there exist a positive integer $\nu$ independent of $x(0)$ and a positive control sequence $\{\boldsymbol{u}(0), \boldsymbol{u}(1), \cdots, \boldsymbol{u}(\nu-1)\}$ which will bring the system to the zero state from 
$\boldsymbol{x}(0)$.

Definition 3 : Let

$$
\begin{aligned}
& \boldsymbol{B} \triangleq\left[\boldsymbol{b}_{1}, \boldsymbol{b}_{2}, \cdots, \boldsymbol{b}_{m}\right] \\
& \boldsymbol{b}_{i} \in R^{n} ; i=1,2, \cdots, m
\end{aligned}
$$

Then we use the following notations:

$$
\begin{aligned}
& \mathrm{L}^{+}[\boldsymbol{B}] \triangleq\left\{\boldsymbol{x} \mid \boldsymbol{x}=c_{1} \boldsymbol{b}_{1}+c_{2} \boldsymbol{b}_{2}+\cdots+c_{m} \boldsymbol{b}_{m} ;\right. \\
& \left.\quad c_{i} \geqq 0 ; i=1,2, \cdots, m\right\} \\
& \langle\boldsymbol{A}, \boldsymbol{B}, N\rangle \triangleq\left[\boldsymbol{B}, \boldsymbol{A} \boldsymbol{B}, \cdots, \boldsymbol{A}^{N-1} \boldsymbol{B}\right] \in R^{n \times N m} \\
& \boldsymbol{E}_{m} \triangleq[1,1, \cdots, 1]^{T} \in R^{m}
\end{aligned}
$$

where $T$ denotes the transpose. Furthermore, we use $\boldsymbol{I}_{n}$ as the $n \times n$ identity matrix.

Definition 4: If $x_{i}>0$ or $x_{i} \geqq 0$ for all $i=1,2, \cdots, n$, then $\boldsymbol{x}=\left[x_{1}, x_{2}, \cdots, x_{n}\right]^{T}$ is called to be a positive $(\boldsymbol{x}>$ 0 ) or nonnegative vector ( $\boldsymbol{x} \geqq \mathbf{0}$ ), respectively.

Definition 5 : Let $\boldsymbol{A}$ and $\boldsymbol{B}$ be transformed into

$$
\begin{aligned}
& \boldsymbol{A}=\left[\begin{array}{cc}
\tilde{\boldsymbol{A}}_{11} & \tilde{\boldsymbol{A}}_{12} \\
\mathbf{0} & \tilde{\boldsymbol{A}}_{22}
\end{array}\right], \boldsymbol{B}=\left[\begin{array}{c}
\widetilde{\boldsymbol{B}}_{1} \\
\widetilde{\boldsymbol{B}}_{2}
\end{array}\right], \\
& \tilde{\boldsymbol{A}} \tilde{\boldsymbol{B}}_{11} \in R^{\tilde{n}_{1} \times \tilde{n}_{1}}, \tilde{\boldsymbol{A}}_{12} \in R^{\tilde{n}_{1} \times \tilde{n}_{2}}, \tilde{\boldsymbol{A}}_{22} \in R^{\tilde{n}_{2} \times \tilde{n}_{2}}, \\
& \tilde{\boldsymbol{B}}_{1} \in R^{\tilde{n}_{1} \times m}, \tilde{\boldsymbol{B}}_{2} \in R^{\tilde{n}_{2} \times m}, \\
& n=\tilde{n}_{1}+\tilde{n}_{2}
\end{aligned}
$$

by a nonsinngular transformation of the state variable. Then system $\tilde{S}$ described by

$$
\tilde{S}: \tilde{\boldsymbol{x}}(k+1)=\tilde{\boldsymbol{A}}_{22} \tilde{\boldsymbol{x}}(k)+\widetilde{\boldsymbol{B}}_{2} \boldsymbol{u}(k)
$$

where $\tilde{\boldsymbol{x}}(k) \in R^{\tilde{n}_{2}}$ is called the subsystem of $S$.

\section{Positive Controllability}

It is clear that the positive controllability is invariant under any nonsingular transformation of the state variable. Thus, system $S$ described by ( 1 ) can be decomposed into the following two subsystems ${ }^{2}$ :

$$
\begin{aligned}
& S_{o}: \boldsymbol{x}_{o}(k+1)=\boldsymbol{A}_{o} \boldsymbol{x}_{o}(k)+\boldsymbol{B}_{o} \boldsymbol{u}(k) \\
& S_{r}: \boldsymbol{x}_{r}(k+1)=\boldsymbol{A}_{r} \boldsymbol{x}_{r}(k)+\boldsymbol{B}_{r} \boldsymbol{u}(k)
\end{aligned}
$$

where $\boldsymbol{A}_{o}$ is a nilpotent matrix, and $\boldsymbol{A}_{r}$ is a nonsingular matrix ;

$$
\begin{aligned}
& \boldsymbol{A}_{o} \in R^{n_{o} \times n_{o}}, \quad \boldsymbol{B}_{o} \in R^{n_{o} \times m}, \boldsymbol{x}_{o}(k) \in R^{n_{o}}, \\
& \boldsymbol{A}_{r} \in R^{n_{r \times n_{r}}}, \boldsymbol{B}_{r} \in R^{n_{r \times m}}, \boldsymbol{x}_{r}(k) \in R^{n_{r}}, \\
& n=n_{o}+n_{r}
\end{aligned}
$$

Now we obtain the following theorem.

Theorem 1 : System $S$ is positive controllable if and only if subsystem $S_{r}$ is positive controllable. $\square$ (Proof) Necessity: It is clear that if subsystem $S_{r}$ is not positive controllable, then system $S$ is not positive controllable. Hence the necessity is established.

Sufficiency : If subsystem $S_{r}$ is positive controllable, then by Deffinition 2, there exist a positive integer $\nu_{r}$ and a positive control sequence $\left\{\boldsymbol{u}(0), \boldsymbol{u}(1), \cdots, \boldsymbol{u}\left(\nu_{r}\right.\right.$
$-1)\}$ which will bring subsystem $S_{r}$ to the zero state $\boldsymbol{x}_{r}\left(\nu_{r}\right)=\mathbf{0}$ for any initial state $\boldsymbol{x}_{r}(0)$. Applying the positive control sequence $\left\{\boldsymbol{u}(0), \boldsymbol{u}(1), \cdots, \boldsymbol{u}\left(\nu_{r}-1\right), \mathbf{0}, \mathbf{0}, \cdots\right.$, $0\}$ with length $\nu_{r}+n_{o}$, to subsystems $S_{o}$ and $S_{r}$ leads to $\boldsymbol{x}_{o}\left(\nu_{r}+n_{o}\right)=\mathbf{0}$ and $\boldsymbol{x}_{r}\left(\nu_{r}+n_{o}\right)=\mathbf{0}$, for any initial states $x_{o}(0)$ and $x_{r}(0)$. This means that $S$ is positive controllable by definition 2 . This completes the sufficiency.

Q.E. D.

From Theorem 1, the positive controllability of $S$ may be reduced to that of $S_{r}$.

Theorem 2: System $S_{r}$ is positive controllable if and only if there exists a finite positive integer $N$, such that

$$
\mathrm{L}^{+}\left[\left\langle\boldsymbol{A}_{r}, \boldsymbol{B}_{r}, N\right\rangle\right]=R^{n_{r}}
$$

where

$$
\begin{aligned}
& \boldsymbol{B}_{r} \triangleq\left[\boldsymbol{b}_{r 1}, \boldsymbol{b}_{r 2}, \cdots, \boldsymbol{b}_{r m}\right] \\
& \boldsymbol{b}_{r i} \in R^{n_{r}} ; i=1,2, \cdots, m
\end{aligned}
$$

(Proof) Suppose that $x_{r}(N)=0$ is realized by applying a positive control sequence $\{\boldsymbol{u}(0), \boldsymbol{u}(1), \cdots, \boldsymbol{u}(N$ $-1)\}$ to system $S_{r}$. Then we get

$$
\begin{aligned}
& \boldsymbol{A}_{r}^{N} \boldsymbol{x}_{r}(0)+\boldsymbol{A}_{r}^{N-1} \boldsymbol{B}_{r} \boldsymbol{u}(0) \\
& \quad+\boldsymbol{A}_{r}^{N-2} \boldsymbol{B}_{r} \boldsymbol{u}(1)+\cdots+\boldsymbol{B}_{r} \boldsymbol{u}(N-1)=\mathbf{0}
\end{aligned}
$$

Two $n_{r}$ dimensional vectors, $\boldsymbol{A}_{r}^{N} \boldsymbol{x}_{r}(0)$ and $\boldsymbol{x}_{r}(0)$, correspond to each other, since $\boldsymbol{A}_{r}^{N}$ is nonsingular. Thus it is possible to choose an appropreate positive control sequence $\{\boldsymbol{u}(0), \boldsymbol{u}(1), \cdots, \boldsymbol{u}(N-1)\}$ such that the left side of ( 8 ) may be zero for any initial state $\boldsymbol{x}_{r}(0)$, if and only if ( 7 ) holds.

Therfore, by definition 2 , Theorem 2 is established.

Theorem 2 gives a geometrical interpretation of a positive controllable system.

The following two lemmas can be directly obtained from Theorem 2 and Definition 3.

Lemma 1: If system $S_{r}$ is positive controllable, then for any $x \in R^{n_{r}}$ there exist a positive integer $N$ independent of $x$ and a nonnegative vector $\boldsymbol{\Omega}$ such that

$$
\left\langle\boldsymbol{A}_{r}, \boldsymbol{B}_{r}, N\right\rangle \boldsymbol{\Omega}=\boldsymbol{x} ; \boldsymbol{\Omega} \geqq \mathbf{0}
$$

Lemma 2: If system $S_{r}$ is positive controllable, then the following two conditions hold:

(1) $S_{r}$ is controllable without input constraints. That is

$$
\operatorname{rank}\left\langle\boldsymbol{A}_{r}, \boldsymbol{B}_{r}, n_{r}\right\rangle=n_{r}
$$

(2) any subsystem of $S_{r}$ is positive controllable. 
Remark 1: Assume that system $S_{r}$ is positive controllable. And let

$$
n_{r}^{*} \triangleq \min \left\{n^{*} \mid \operatorname{rank}\left\langle\boldsymbol{A}_{r}, \boldsymbol{B}_{r}, n^{*}\right\rangle=n_{r}\right\}
$$

Then from Cayley-Hamilton theorem it follows that $1 \leqq n_{r}^{*} \leqq n_{r}$.

Lemma 3 : If there exists a finite positive integer $N$ which satisfies ( 7 ), then

$\operatorname{rank}\left\langle\boldsymbol{A}_{r}, \boldsymbol{B}_{r}, N\right\rangle=n_{r}$

(Proof) If $\operatorname{rank}\left\langle\boldsymbol{A}_{r}, \boldsymbol{B}_{r}, N\right\rangle \neq n_{r}$ for some integer $N$, then the range space of $\left\langle\boldsymbol{A}_{r}, \boldsymbol{B}_{r}, N\right\rangle$ which contains $\mathrm{L}^{+}\left[\left\langle\boldsymbol{A}_{r}, \boldsymbol{B}_{r}, N\right\rangle\right]$ is properly included by $R^{n_{r}}$. Hence, ( 7 ) does not hold.

Therefore, Lemma 3 is established.

Q.E. D.

Remark 2: The positive integer $N$ in Lemma 3 and $n_{r}^{*}$ in Remark 1 satisfy $n_{r}^{*} \leqq N$.

Next, we let

$$
\begin{array}{r}
\left\langle\boldsymbol{A}_{r}, \boldsymbol{B}_{r}, N\right\rangle \triangleq\left[\boldsymbol{e}_{1}, \boldsymbol{e}_{2}, \cdots, \boldsymbol{e}_{N m}\right], \\
\boldsymbol{e}_{m j+i} \triangleq \boldsymbol{A}_{r}^{j} \boldsymbol{b}_{i} \in R^{n_{r}} ; i=1,2, \cdots, m ; \\
j=0,1, \cdots, N-1
\end{array}
$$

Then we obtain the following Theorem 3 .

Theorem 3 : System $S_{r}$ is positive controllable if and only if there exist positive integers $\left\{N, h, K_{1}, K_{2}\right.$, $\left.\cdots, K_{h}\right\}$ and positive scalars $\left\{c_{K_{1}}, c_{K_{2}}, \cdots, c_{K_{h}}\right\}$ which satisfy the following three conditions;

(1) $c_{K_{1}} \boldsymbol{e}_{K_{1}}+c_{K_{2}} \boldsymbol{e}_{K_{2}}+\cdots+c_{K_{h}} \boldsymbol{e}_{K_{h}}=\mathbf{0}$

(2) $1 \leqq K_{1}<K_{\dot{2}}<\cdots<K_{h} \leqq N m$

(3) $\operatorname{rank}\left[\boldsymbol{e}_{K_{1}}, \boldsymbol{e}_{K_{2}}, \cdots, \boldsymbol{e}_{K_{h}}\right]=n_{r}<h \leqq N m$

The proof is given in Appendix A.

It is possible to determine whether a given system $S_{r}$ is positive controllable or not by using Theorem 3 .

Lemma 4 : Suppose that

$$
\operatorname{rank}\left\langle\boldsymbol{A}_{r}, \boldsymbol{B}_{r}, n_{r}\right\rangle=n_{r}
$$

Then, system $S_{r}$ is positive controllable, if and only if there exist a positive integer $N$ and a nonnegative vector $\boldsymbol{\Omega}$ such that

$$
\left\langle\boldsymbol{A}_{r}, \boldsymbol{B}_{r}, N 》 \boldsymbol{\Omega}=\mathbf{0}, \boldsymbol{\Omega} \geqq \mathbf{0}\right.
$$

where

$$
\begin{aligned}
& \boldsymbol{\Omega} \triangleq\left[\boldsymbol{\omega}_{1}^{T}, \boldsymbol{\omega}_{2}^{T}, \cdots, \boldsymbol{\omega}_{N}^{T}\right]^{T}, \boldsymbol{\omega}_{1}>\mathbf{0} \\
& \boldsymbol{\omega}_{i} \in R^{m} ; i=1,2, \cdots, N
\end{aligned}
$$

(Proof) Necessity: If $S_{r}$ is positive controllable, then from Theorem 3, (12) (14) hold.

Thus from (14), we have

$$
\boldsymbol{e}_{i}=d_{i 1} \boldsymbol{e}_{K_{1}}+d_{i 2} \boldsymbol{e}_{K_{2}}+\cdots+d_{i h} \boldsymbol{e}_{K_{h}} ; i=1,2, \cdots, m
$$

where $d_{i j}$ 's $(i=1,2, \cdots, m ; j=1,2, \cdots, h)$ are appropreate scalars. Premultiplying both sides of (12) by a sufficiently large positive scalar $M$ and adding (19) yield

$$
\boldsymbol{e}_{1}+\boldsymbol{e}_{2}+\cdots+\boldsymbol{e}_{m}+c_{K_{1}}^{*} \boldsymbol{e}_{K_{1}}+c_{K_{2}}^{*} \boldsymbol{e}_{K_{2}}+\cdots+c_{K_{h}}^{*} \boldsymbol{e}_{K_{h}}=0
$$

where

$$
c_{K_{i}}^{*}=M c_{K_{i}}-d_{1 i}-d_{2 i}-\cdots-d_{m i}>0 ; i=1,2, \cdots, h
$$

It can be shown that (20) and (21) are equivalent to (16) $\sim(18)$.

Sufficiency : From (16) and (17) we get

$$
\boldsymbol{B}_{r} \boldsymbol{\omega}_{1}+\boldsymbol{A}_{r} \boldsymbol{B}_{r} \boldsymbol{\omega}_{2}+\cdots+\boldsymbol{A}_{r}^{N-1} \boldsymbol{B}_{r} \boldsymbol{\omega}_{N}=\mathbf{0}
$$

Premultiplying (22) by $\boldsymbol{A}_{r}, \boldsymbol{A}_{r}^{2}, \cdots, \boldsymbol{A}_{r}^{n_{r-1}}$ and adding these equations yield

$$
\left\langle\boldsymbol{A}_{r}, \boldsymbol{B}_{r}, n_{r}+N-1\right\rangle \boldsymbol{\Omega}^{+}=\mathbf{0}, \boldsymbol{\Omega}^{+} \geqq \mathbf{0}
$$

where

$$
\begin{aligned}
& \boldsymbol{\Omega}^{+} \triangleq\left[\boldsymbol{\omega}_{1}^{+T}, \boldsymbol{\omega}_{2}^{+T}, \cdots, \boldsymbol{\omega}_{n_{r}+N-1}^{+}\right]^{T}, \boldsymbol{\omega}_{1}^{+}>\mathbf{0}, \\
& \boldsymbol{\omega}_{i}^{+}=\boldsymbol{\omega}_{1}+\boldsymbol{\omega}_{2}+\cdots+\boldsymbol{\omega}_{i}>\mathbf{0} ; i=1,2, \cdots, n_{r}, \\
& \boldsymbol{\omega}_{i}^{+} \geqq \mathbf{0} ; i=n_{r}+1, n_{r}+2, \cdots, n_{r}+N-1
\end{aligned}
$$

It is easy to see that (23) and (24) are equivalent to (12) $\sim(14)$ by replacing $n_{r}+N$ by $N$.

Hence, system $S_{r}$ is positive controllable by Theorem 3 .

Q.E. D.

Lemma 5: System $S_{r}$ is positive controllable if and only if for any samll $\varepsilon$, there exist positive integers $\left\{N, h, K_{1}, K_{2}, \cdots, K_{h}\right\}$ independent of $\varepsilon$ and positive scalars $\left\{c_{K_{1}}, c_{K_{2}}, \cdots, c_{K_{h}}\right\}$ which satisfy the following three conditions :

(1) $c_{K_{1}} \boldsymbol{e}_{K_{1}}+c_{K_{2}} \boldsymbol{e}_{K_{2}}+\cdots+c_{K_{h}} \boldsymbol{e}_{K_{h}}=\boldsymbol{\varepsilon}$

(2) $1 \leqq K_{1}<K_{2}<\cdots<K_{h} \leqq N m$

(3) $\operatorname{rank}\left[\boldsymbol{e}_{K_{1}}, \boldsymbol{e}_{K_{2}}, \cdots, \boldsymbol{e}_{K_{h}}\right]=n_{r}<h \leqq N m$

where $\varepsilon \triangleq\left[\varepsilon_{1}, \varepsilon_{2}, \cdots, \varepsilon_{n_{r}}\right]^{T}$, and the norm

$$
\|\varepsilon\| \triangleq \sqrt{\varepsilon_{1}^{2}+\varepsilon_{2}^{2}+\cdots+\varepsilon_{n_{r}}^{2}}>0
$$

is sufficiently small.

(Proof) Sufficiency: We suppose (25) (28) hold. Then we can select $n_{r}$ independent vectors $\left\{\boldsymbol{e}_{W_{1}}, \boldsymbol{e}_{W_{2}}\right.$, $\left.\cdots, \boldsymbol{e}_{W_{n}}\right\}$ out of $h$ vectors $\left\{\boldsymbol{e}_{K_{1}}, \boldsymbol{e}_{K_{2}}, \cdots, \boldsymbol{e}_{K_{h}}\right\}$ in (27). Thus, there exist $n_{r}$ scalars $\left\{\alpha_{W_{1}}^{*}, \alpha_{W_{2}}^{*}, \cdots, \alpha_{W_{r}}^{*}\right\}$, such that

$$
\alpha_{W_{1}}^{*} \boldsymbol{e}_{W_{1}}+\alpha_{W_{2}}^{*} \boldsymbol{e}_{W_{2}}+\cdots+\alpha_{W_{n}}^{*} \boldsymbol{e}_{W_{n_{r}}}=-\boldsymbol{\varepsilon}
$$

Here $\alpha_{W_{i}}^{*} \mathrm{~s}\left(i=1,2, \cdots, n_{r}\right)$ are sufficiently small scalars, since $\|\varepsilon\|$ is sufficiently small. Adding (25) and (29), we obtain

$$
c_{K_{1}}^{*} e_{K_{1}}+c_{K_{2}}^{*} e_{K_{2}}^{*}+\cdots+c_{K_{h}}^{*} e_{K_{h}}=0
$$

where

$$
c_{i}^{*}=c_{i}>0 ; i=K_{1}, K_{2}, \cdots, K_{h} \text { and }
$$




$$
\begin{gathered}
\quad i \neq W_{1}, W_{2}, \cdots, W_{n_{r}} \\
c_{i}^{*}=c_{i}+\alpha_{t}^{*}>0 ; \quad i=W_{1}, W_{2}, \cdots, W_{n_{r}}
\end{gathered}
$$

Thus from (30), (26) and (27), $S_{r}$ is poositive controllable by virtue of Theorem 3 .

Necessity : If system $S_{r}$ is positive controllable, then by Theorem 3 there exist positive integers $\{N, h$, $\left.K_{1}, K_{2}, \cdots, K_{h}\right\}$ independent of $\varepsilon$ and positive scalars $\left\{c_{K 1}, c_{K 2}, \cdots, c_{K_{n}}\right\}$ which satisfy $(12) \sim(14)$. Thus, we have

$$
\alpha_{W_{1}}^{*} e_{W_{1}}+\alpha_{W_{2}}^{*} e_{W_{2}}+\cdots+\alpha_{W_{r}}^{*} \boldsymbol{e}_{W_{n_{r}}}=\boldsymbol{\varepsilon}
$$

where $\alpha w_{W_{t}}\left(i=1,2, \cdots, n_{r}\right)$ are sufficiently small scalars. By a similar way discussed in the sufficiency, we obtain

$$
\begin{aligned}
& c_{K_{1}}^{*} e_{K_{1}}+c_{K_{2}}^{*} \boldsymbol{e}_{K_{2}}+\cdots+c_{K_{h}}^{*} \boldsymbol{e}_{K_{h}}=\varepsilon \\
& c_{i}^{*}>0 ; i=K_{1}, K_{2}, \cdots, K_{h}
\end{aligned}
$$

Replacing $c_{i}^{*}$ by $c_{i}$, we have (25), (26) and (27)

Lemma 6: Suppose that

$$
\operatorname{rank}\left\langle\boldsymbol{A}_{r}, \boldsymbol{B}_{r}, n_{r}\right\rangle=n_{r}
$$

Then, system $S_{r}$ is positive controllable, if and only if for any small $\varepsilon$ there exist a positive integer $N$ independent of $\varepsilon$ and a nonnegative vector $\boldsymbol{\Omega}$ such that

$$
\left\langle A_{r}, B_{r}, N\right\rangle \boldsymbol{\Omega}=\boldsymbol{\varepsilon} ; \boldsymbol{\Omega} \geqq \mathbf{0}
$$

where

$$
\begin{aligned}
& \boldsymbol{\Omega} \triangleq\left[\boldsymbol{\omega}_{1}^{T}, \boldsymbol{\omega}_{2}^{T}, \cdots, \boldsymbol{\omega}_{N}^{T}\right]^{T}, \boldsymbol{\omega}_{1}>\mathbf{0} \\
& \boldsymbol{\omega}_{i} \in R^{m} ; i=1,2, \cdots, N
\end{aligned}
$$

(Proof) Necessity: If $S_{r}$ is positive controllable, then from Lemma 4, (16) (18) hold. We can introduce (37) (39) by modifying slightly the proof of Lemma 5.

Sufficiency: We assume that $(36) \sim(39)$ hold. Then premultiplying (37) by $\boldsymbol{I}, \boldsymbol{A}_{r}, \boldsymbol{A}_{r}^{2}, \cdots, \boldsymbol{A}_{r}^{n_{r-1}}$ and adding these equatinos yield

$$
\begin{array}{r}
\left\langle\boldsymbol{A}_{r}, \boldsymbol{B}_{r}, n_{r}+N-1\right\rangle \boldsymbol{\Omega}^{+} \\
\quad=\left(\boldsymbol{I}+\boldsymbol{A}_{r}+\cdots+\boldsymbol{A}_{r}^{n_{r-1}}\right) \boldsymbol{\varepsilon}
\end{array}
$$

where $\boldsymbol{\Omega}^{+}$is given in (24).

Since $\varepsilon$ is sufficiently small, the right side of $(40)$ is sufficiently small. It is easy to see that (40) and (24) are equivalent to $(25) \sim(27)$ by replacing $n_{r}+N$ by $N$. Hence by Lemma 5, system $S_{r}$ is positive controllable.

Q.E.D.

In order to investigate the structure of positive controllable systems, we decompose system $S_{r}$ into the following two subsystems $S_{t}$ and $S_{q}$, considering the eigenvalues.

$$
\begin{aligned}
& S_{t}: \boldsymbol{x}_{t}(k+1)=\boldsymbol{A}_{t} \boldsymbol{x}_{t}(k)+\boldsymbol{B}_{t} \boldsymbol{u}(k) \\
& S_{q}: \boldsymbol{x}_{q}(k+1)=\boldsymbol{A}_{q} \boldsymbol{x}_{q}(k)+\boldsymbol{B}_{q} \boldsymbol{u}(k)
\end{aligned}
$$

where

$$
\begin{aligned}
& \boldsymbol{A}_{t} \in R^{n_{t} \times n_{t}}, \boldsymbol{B}_{t} \in R^{n_{t} \times m}, \boldsymbol{x}_{t}(k) \in R^{n_{t}}, \\
& \boldsymbol{A}_{q} \in R^{n_{q} \times n_{q}}, \boldsymbol{B}_{q} \in R^{n_{q} \times m}, \boldsymbol{x}_{q}(k) \in R^{n_{q}}, \\
& n_{r}=n_{t}+n_{q}, \\
& \lambda_{i}\left(\boldsymbol{A}_{t}\right)<0 \text { or } \operatorname{Im}\left\{\lambda_{i}\left(\boldsymbol{A}_{t}\right)\right\} \neq 0 ; i=1,2, \cdots, n_{t}, \\
& \lambda_{i}\left(\boldsymbol{A}_{q}\right)>0 ; i=1,2, \cdots, n_{q}
\end{aligned}
$$

and $\lambda_{i}(\boldsymbol{A})$ denotes the $i$-th eigenvalue of $\boldsymbol{A}$.

Then we may establish the following theorem.

Theorem 4: System $S_{r}$ is positive controllable if and only if the following two conditions hold:

(1) $\operatorname{rank}\left\langle\boldsymbol{A}_{r}, \boldsymbol{B}_{r}, n_{r}\right\rangle=n_{r}$

(2) system $S_{q}$ is positive controllable.

The proof is given in Appendix B.

From Theorem 4, the controllability of $S_{r}$ may be reduced to that of $S_{q}$.

Next, if system $S_{q}$ has more than two distinct eigenvalues, then we can decompose system $S_{q}$ into the following two subsystems $S_{a}$ and $S_{b}$,

$$
\begin{aligned}
& S_{a}: \boldsymbol{x}_{a}(k+1)=\boldsymbol{A}_{a} \boldsymbol{x}_{a}(k)+\boldsymbol{B}_{a} \boldsymbol{u}(k) \\
& S_{b}: \boldsymbol{x}_{b}(k+1)=\boldsymbol{A}_{b} \boldsymbol{x}_{b}(k)+\boldsymbol{B}_{b} \boldsymbol{u}(k)
\end{aligned}
$$

where

$$
\begin{aligned}
& \boldsymbol{A}_{a} \in R^{n_{a} \times n_{a}}, \quad \boldsymbol{B}_{a} \in R^{n_{a} \times m}, x_{a}(k) \in R^{n_{a}}, \\
& \boldsymbol{A}_{b} \in R^{n_{b} \times n_{b}}, \boldsymbol{B}_{b} \in R^{n_{b} \times m}, \boldsymbol{x}_{b}(k) \in R^{n_{b}}, \\
& n_{q}=n_{a}+n_{b}, \\
& 0<\lambda_{i}\left(\boldsymbol{A}_{a}\right)<\lambda_{j}\left(\boldsymbol{A}_{b}\right) ; i=1,2, \cdots, n_{a} ; \\
& \quad j=1,2, \cdots, n_{b}
\end{aligned}
$$

Then we have the following theorem.

Theorem 5: System $S_{q}$ is positive controllable, if and only if the following three conditions hold

(1) $\operatorname{rank}\left\langle\boldsymbol{A}_{q}, \boldsymbol{B}_{q}, n_{q}\right\rangle=n_{q}$

(2) system $S_{a}$ is positive controllable.

(3) system $S_{b}$ is positive controllable.

The proof is given in Appendix C.

From Theorem 5, the controllability of system $S_{q}$ is reduced to those of its subsystems $S_{a}$ and $S_{b}$.

Furthermore, if system $S_{q}$ has $Q$ distinct positive eigenvalues, then we can transform system $S_{q}$ into the following Jordan canonical form:

$$
\begin{aligned}
& \boldsymbol{A}_{q}=\operatorname{diag}\left[\boldsymbol{A}_{1}, \boldsymbol{A}_{2}, \cdots, \boldsymbol{A}_{Q}\right] \in R^{n_{q} \times n_{q}}, \\
& \boldsymbol{B}_{q}=\left[\boldsymbol{B}_{1}^{T}, \boldsymbol{B}_{2}^{T}, \cdots, \boldsymbol{B}_{Q}^{T}\right]^{T} \in R^{n_{q} \times m}, \\
& \boldsymbol{A}_{i}=\operatorname{diag}\left[\boldsymbol{A}_{i 1}, \boldsymbol{A}_{i 2}, \cdots, \boldsymbol{A}_{i r(i)}\right] \in R^{n_{i} \times n_{i}}, \\
& \boldsymbol{B}_{i}=\left[\boldsymbol{B}_{i 1}^{T}, \boldsymbol{B}_{i 2}^{T}, \cdots, \boldsymbol{B}_{i r(i)}^{T}\right]^{T} \in R^{n_{i} \times m}, \\
& n_{1}+n_{2}+\cdots+n_{Q}=n_{q},
\end{aligned}
$$




$$
\begin{aligned}
& \boldsymbol{A}_{i j}=\boldsymbol{J}\left[\lambda_{i}, n_{i j}\right] \in R^{n_{i j} \times n_{i j}}, \\
& \boldsymbol{B}_{i j}=\left[\boldsymbol{b}_{n_{i j i}}^{T}, \cdots, \boldsymbol{b}_{2 i j}^{T}, \boldsymbol{b}_{1 i j}^{T}\right]^{T} \in R^{n_{i j \times m}}, \\
& n_{i 1}+n_{i 2}+\cdots+n_{i r(i)}=n_{i}, \\
& i=1,2, \cdots, Q ; j=1,2, \cdots, r(i)
\end{aligned}
$$

where $J[\lambda, n]$ denotes a Jordan block of order $n$ with eigenvalue $\lambda$. Further we can assume without loss of generality that

$$
\begin{aligned}
& 0<\lambda_{1}<\lambda_{2}<\cdots<\lambda_{Q} \\
& 1 \leqq n_{i 1} \leqq n_{i 2} \leqq \cdots \leqq n_{i r(i)}
\end{aligned}
$$

Then, by using Theorem 5 repeatedly, we can directly obtain the following theorem.

Theorem 6: System $S_{q}$ is positive controllable if and only if system $S_{i}$ described by

$$
S_{i}: \boldsymbol{x}_{i}(k+1)=\boldsymbol{A}_{i} \boldsymbol{x}_{\boldsymbol{i}}(k)+\boldsymbol{B}_{i} \boldsymbol{u}(k)
$$

is positive controllable for each $i=1,2, \cdots, Q$

From Theorem 6 , the positive controllability of a system may be reduced to those of its $Q$ subsystems $S_{i}(i=1,2, \cdots, Q)$ with $Q$ distinct positive eigenvalues. Since the dimension of $S_{i}$ is smaller than that of $S_{r}$, the positive controllability test in Theorem 3 may be simplified very much by using Theorem 7 .

Now, for any positive scalar $\lambda$ and any positive integer $F$, we consider the following system

$$
S^{*}: \boldsymbol{x}^{*}(k+1)=\boldsymbol{A}^{*} \boldsymbol{x}^{*}(k)+\boldsymbol{B}^{*} \boldsymbol{u}(k)
$$

where

$$
A^{*} \triangleq \lambda I_{F}, B^{*} \triangleq\left[\xi_{1}, \xi_{2}, \cdots, \xi_{m}\right] \in R^{F \times m}
$$

Then, we get the following theorem. The proof is straightforward based on Theorem 3 .

Theorem 7: System $S^{*}$ is positive controllalle if and only if there exsist positive integers $\left\{h, K_{1}, K_{2}, \cdots\right.$, $\left.K_{h}\right\}$ and positive scalars $\left\{c_{K_{1}}, c_{K_{2}}, \cdots, c_{K_{h}}\right\}$ which satisfy the following three conditions:

(1) $c_{K_{1}} \boldsymbol{\xi}_{K_{1}}+c_{K_{2}} \boldsymbol{\xi}_{K_{2}}+\cdots+c_{K_{h}} \boldsymbol{\xi}_{K_{h}}=\mathbf{0}$

(2) $1 \leqq K_{1}<K_{2}<\cdots<K_{h} \leqq m$

(3) $\operatorname{rank}\left[\boldsymbol{\xi}_{K_{1}}, \boldsymbol{\xi}_{K_{2}}, \cdots, \boldsymbol{\xi}_{K_{h}}\right]=F<h \leqq m$

Remark 3: In Theorem 7, $F<m$ is necessary for $S^{*}$ to be positive controllable. Thus, a single input system $(m=1)$ having any positive eigenvalues is not positive controllable. This agrees with the former results ${ }^{3}$.

Lemma 7: System $S^{*}$ is positive controllable, if and only if for any $x^{*} \in R^{F}$, there exists a nonnegative vector $\Omega^{*}$, such that

$$
B^{*} \Omega^{*}=x^{*} ; \Omega^{*} \geqq 0
$$

(Proof) Since $A^{*}=\lambda I_{F}$ and $\lambda>0$, it is easy to show that for any positive integer $N \geqq 1$,

$$
\mathrm{L}^{+}\left[\left\langle\boldsymbol{A}^{*}, \boldsymbol{B}^{*}, N\right\rangle\right]=\mathrm{L}^{+}\left[\boldsymbol{B}^{*}\right]
$$

Thus from Theorem 2 and (58), system $S^{*}$ is positive controllable, if and only if

$$
\mathrm{L}^{+}\left[\mathrm{B}^{*}\right]=R^{F}
$$

It is evident that (59) is equivalent to that for any $x^{*} \in R^{F}$ there exists a nonnegative vector $\Omega^{*}$, such that (57) holds.

Therefore, we have Lemma 7.

Q.E.D.

The following lemma will be shown in Appendix D.

Lemma 8: Let $F$ and $G$ be any positive integers. Then, system $S_{c}^{+}$described by

$$
S_{G}^{+}: x^{+}(k+1)=\Phi_{G} x^{+}(k)+\Gamma_{G} u(k)
$$

where

$$
\begin{aligned}
& \boldsymbol{\Phi}_{G} \triangleq\left[\begin{array}{ccccc}
A^{*} & \boldsymbol{I}_{F} & \mathbf{0} & \cdots & \mathbf{0} \\
\mathbf{0} & \boldsymbol{A}^{*} & \boldsymbol{I}_{F} & \cdots & \mathbf{0} \\
& \cdots & \cdots & \cdots & \\
\mathbf{0} & \cdots & \mathbf{0} & \boldsymbol{A}^{*} & \boldsymbol{I}_{F} \\
\mathbf{0} & \cdots & \mathbf{0} & \mathbf{0} & \boldsymbol{A}^{*}
\end{array}\right], \boldsymbol{\Gamma}_{G} \triangleq\left[\begin{array}{c}
\boldsymbol{B}_{G}^{+} \\
\boldsymbol{B}_{G-1}^{+} \\
\cdots \\
\boldsymbol{B}_{2}^{+} \\
\boldsymbol{B}_{1}^{+}
\end{array}\right], \\
& \boldsymbol{\Phi}_{G} \in R^{F G \times F G}, \boldsymbol{\Gamma}_{G} \in R^{F G \times m}, \boldsymbol{B}_{1}^{+}=\boldsymbol{B}^{*} \\
& \boldsymbol{B}_{i}^{+} \in R^{F \times m} ; i=1,2, \cdots, G
\end{aligned}
$$

is positive controllable, if and only if system $S^{*}$ described by (52) is positive controllable.

Finally, we have the following theorem.

Theorem 8: System $S_{q}$ is positive controllable if and only if system $S_{i}^{*}$ described by

$$
S_{i}^{*}: \boldsymbol{x}_{i}^{*}(k+1)=\boldsymbol{A}_{i}^{*} \boldsymbol{x}_{i}^{*}(k)+\boldsymbol{B}_{i}^{*} \boldsymbol{u}(k)
$$

where

$$
\begin{aligned}
& \boldsymbol{A}_{i}^{*} \triangleq \lambda_{i} \boldsymbol{I}_{r(i),} \\
& \boldsymbol{B}_{i}^{*} \triangleq\left[\begin{array}{c}
\boldsymbol{b}_{1 i 1} \\
\boldsymbol{b}_{1 i 2} \\
\ldots \\
\boldsymbol{b}_{1 i r(i)}
\end{array}\right] \in R^{r(i) \times m}
\end{aligned}
$$

is positive controllable for each $i=1,2, \cdots, Q$ (Proof) Necessity: If system $S_{q}$ is positive controllable, then from Theorem 6 , system $S_{i}$ is positive controllable for each $i=1,2, \cdots, Q$.

On the other hand, system $S_{i}^{*}$ is the subsystem of $S_{i}$ from (48) and (64). Therefore, from Lemma 1, system $S_{i}^{*}$ is positive controllable for each $i=1,2, \cdots, Q$.

Sufficiency : For each $i=1,2, \cdots, Q$, consider a system described by

$$
S_{i}^{+}: \boldsymbol{x}_{i}^{+}(k+1)=\boldsymbol{A}_{i}^{+} \boldsymbol{x}_{i}^{+}(k)+\boldsymbol{B}_{i}^{+} \boldsymbol{u}(k)
$$

where 


$$
\begin{aligned}
\boldsymbol{A}_{i}^{+} \triangleq\left[\begin{array}{ccccc}
\boldsymbol{A}_{i}^{*} & \boldsymbol{I}_{r(i)} & \mathbf{0} & \cdots & \mathbf{0} \\
\mathbf{0} & \boldsymbol{A}_{i}^{*} & \boldsymbol{I}_{r(i)} & \cdots & 0 \\
& \cdots & \cdots & \cdots & \\
0 & \cdots & \mathbf{0} & \boldsymbol{A}_{i}^{*} & \boldsymbol{I}_{r(i)} \\
\mathbf{0} & \cdots & \mathbf{0} & \mathbf{0} & \boldsymbol{A}_{i}^{*}
\end{array}\right] \in R^{r(i) G(i) \times r(i) G(i)}, \\
\boldsymbol{B}_{i}^{+} \triangleq\left[\begin{array}{c}
\boldsymbol{B}_{i G(i)}^{+} \\
\cdots \\
\boldsymbol{B}_{i 2}^{+} \\
\boldsymbol{B}_{i 1}^{+}
\end{array}\right] \in R^{r(i) G(i) \times m} \\
\boldsymbol{B}_{i 1}^{+}=\boldsymbol{B}_{i}^{*}, G(i) \triangleq n_{i r(i)}
\end{aligned}
$$

Then it is easy to show that $\boldsymbol{A}_{i}^{+}$and $\boldsymbol{B}_{i}^{+}$can be chosen so that system $S_{i}$ is the subsystem of $S_{i}^{+}$. Now, if system $S_{i}^{*}$ is positive controllable for each $i=1,2, \cdots, Q$, then system $S_{i}^{+}$is positive controllable for each $i=1,2, \cdots, Q$, by Lemma 8 . Since system $S_{i}$ is the subsystem of $S_{i}^{+}$, system $S_{i}$ is positive controllable, by Lemma 2 . Therefore, by Theorem 6 , system $S_{q}$ is positive controllable.

Q. E. D.

Ultimately, the controllability test may be checked by using Theorem 7 and Theorem 8 , which are the main feachures of this work.

\section{An Example}

Consider a system represented by

$$
\boldsymbol{A}=\left[\begin{array}{rrrrr}
0 & 0 & 0 & 0 & 0 \\
0 & -1 & 0 & 0 & 0 \\
0 & 0 & 3 & 0 & 0 \\
0 & 0 & 0 & 3 & 1 \\
0 & 0 & 0 & 0 & 3
\end{array}\right], \boldsymbol{B}=\left[\begin{array}{rrr}
2 & 1 & 0 \\
1 & 2 & 3 \\
1 & -2 & 1 \\
0 & 3 & 4 \\
-1 & 0 & 2
\end{array}\right]
$$

where $n=5, m=3$. Then we have

$$
\begin{aligned}
\boldsymbol{A}_{o}= & 0, \boldsymbol{B}_{o}=\left[\begin{array}{lll}
2 & 1 & 0
\end{array}\right] \\
\boldsymbol{A}_{r} & =\left[\begin{array}{rrrr}
-1 & 0 & 0 & 0 \\
0 & 3 & 0 & 0 \\
0 & 0 & 3 & 1 \\
0 & 0 & 0 & 3
\end{array}\right], \boldsymbol{B}_{r}=\left[\begin{array}{rrr}
1 & 2 & 3 \\
1 & -2 & 1 \\
0 & 3 & 4 \\
-1 & 0 & 2
\end{array}\right]
\end{aligned}
$$

where $n_{o}=1, n_{r}=4$,

$$
\operatorname{rank}\left[\boldsymbol{B}_{r}, \boldsymbol{A}_{r} \boldsymbol{B}_{r}, \boldsymbol{A}_{r}^{2} \boldsymbol{B}_{r}, \boldsymbol{A}_{r}^{3} \boldsymbol{B}_{r}\right]=4
$$

Further, we obtain

$$
\begin{aligned}
\boldsymbol{A}_{t} & =-1, \boldsymbol{B}_{t}=\left[\begin{array}{lll}
1 & 2 & 3
\end{array}\right] \\
\boldsymbol{A}_{q} & =\left[\begin{array}{lll}
3 & 0 & 0 \\
0 & 3 & 1 \\
0 & 0 & 3
\end{array}\right], \boldsymbol{B}_{q}=\left[\begin{array}{rrr}
1 & -2 & 1 \\
0 & 3 & 4 \\
-1 & 0 & 2
\end{array}\right]
\end{aligned}
$$

where $n_{t}=1, n_{q}=3$. Thus, we get

$$
\boldsymbol{A}_{1}=\boldsymbol{A}_{q}, \boldsymbol{B}_{1}=\boldsymbol{B}_{q}, Q=1, \lambda_{1}=3, n_{1}=3
$$

Next, we have

$$
\boldsymbol{A}_{11}=3, \boldsymbol{B}_{11}=\left[\begin{array}{lll}
1 & -2 & 1
\end{array}\right] \boldsymbol{b}_{111}
$$

$$
\boldsymbol{A}_{12}=\left[\begin{array}{ll}
3 & 1 \\
0 & 3
\end{array}\right], \quad \boldsymbol{B}_{22}=\left[\begin{array}{rrr}
0 & 3 & 4 \\
-1 & 0 & 2
\end{array}\right] \boldsymbol{b}_{212}
$$

where

$$
n_{11}=1, n_{12}=2, r(1)=2, G(1)=2
$$

Finally, we obtain

$$
S_{1}^{*}: \boldsymbol{x}_{1}^{*}(k+1)=\boldsymbol{A}_{1}^{*} \boldsymbol{x}_{1}^{*}(k)+\boldsymbol{B}_{1}^{*} \boldsymbol{u}(k)
$$

where

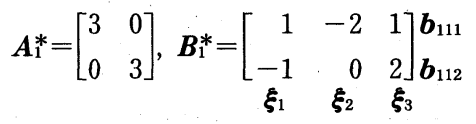

It is easy to verify that

$$
\begin{aligned}
& \operatorname{rank}\left[\boldsymbol{\xi}_{1}, \boldsymbol{\xi}_{2}, \boldsymbol{\xi}_{3}\right]=2 \\
& 4 \boldsymbol{\xi}_{1}+3 \xi_{2}+2 \xi_{3}=\mathbf{0}
\end{aligned}
$$

Thus system $S_{1}^{*}$ is positive controllable from Theorem 7. Then, system $S_{q}$ is positive controllable from Theorem 8. Accordingly, system $S_{r}$ is positive controllable from Theorem 4. Hence, system $S$ is positive controllable from Theorem 1 .

\section{Conclusions}

A necessary and sufficient condition for the controllability to the zero state by positive controls of multiple input discrete-time linear systems is presented based on the Jordan canonical form. The results in this paper reveale the structure of the controllable system with positive controls, and are considered to be fundamental and essensial in the control system theory.

\section{References}

1) R. E. Kalman, Y.C. Ho and K. S. Narendra : Controllability of Linear Dynamical Systems, Contributions to J. Differential Equations, 1, 189/213 (1962)

2) S. Kodama : Controllability of Linear Discrete-Time Systems with Input and State Variable Constraints, J. Inst. Elec. Comm. Engrs. (Japan), 48-5, 884/891 (1965)

3) S. H. Saperstone and J. A. Yorke: Controllability of Linear Oscillatory Systems Using Positive Controls, SIAM J. Contr., 9-2, 253/262 (1971)

4) R. F. Bramer: Controllability in Linear Autonomous Systems with Positive Controllers, SIAM J. Contr., 10 $-2,339 / 353$ (1972)

5) S. H. Saperstone: Global Controllability of Linear systems with Positive Controls, SIAM J. Contr., 11-3, 417/423 (1973)

6) M. E. Evance and D. N. P. Murthy : Controlability of Discrete-Time Systems wth Positive Controls, IEEE Trans., AC-22-6, 942/945 (1977)

7) H. Yoshida : Controllability of Linear Discrete-Time Systems with Positive Controls, Trans. of SICE, 28-5, 646/648 (1992) 


\section{《Appendix A》}

\section{Proof of Theorem 3}

Sufficiency: We can select $n_{r}$ independent vectors $\left\{\boldsymbol{e}_{W_{1}}, \boldsymbol{e}_{W_{2}}, \cdots, \boldsymbol{e}_{W_{n}}\right\}$ out of $h$ vectors $\left\{\boldsymbol{e}_{K_{1}}, \boldsymbol{e}_{K_{2}}, \cdots, \boldsymbol{e}_{K_{h}}\right\}$ in (14), where

$$
1 \leqq W_{1}<W_{2}<\cdots<W_{n_{r}} \leqq N m
$$

Let $\boldsymbol{x}_{r}(0) \in R^{n_{r}}$ be any initial state. Then, $-\boldsymbol{A}_{r}^{N} \boldsymbol{x}_{r}(0) \in R^{n_{r}}$. Thus $-\boldsymbol{A}_{r}^{N} \boldsymbol{x}_{r}(0)$ can be expressed as a linear combination of $n_{r}$ independent vectors $\left\{\boldsymbol{e}_{W_{1},}\right.$, $\left.\boldsymbol{e}_{W_{2}}, \cdots, \boldsymbol{e}_{W_{n}}\right\}$ with real coefficients $\left\{\alpha_{W_{1}}, \alpha_{W_{2}}, \cdots, \alpha_{W_{n}}\right\}$; that is

$$
\alpha_{W_{1}} \boldsymbol{e}_{W_{1}}+\alpha_{W_{2}} \boldsymbol{e}_{W_{2}}+\cdots+\alpha_{W_{r r}} \boldsymbol{e}_{W_{n_{r}}}=-\boldsymbol{A}_{r}^{N} \boldsymbol{x}_{r}(0)
$$

Premultiplying both sides of (12) by a sufficiently large positive scalar $M$ and adding (A.2) yield

$$
\beta_{1} \boldsymbol{e}_{1}+\beta_{2} \boldsymbol{e}_{2}+\cdots+\beta_{N m} \boldsymbol{e}_{N m}=-\boldsymbol{A}_{r}^{N} \boldsymbol{x}_{r}(0)
$$

where

$$
\begin{aligned}
& \beta_{i}=0 ; i=1,2, \cdots, N m \text { and } i \neq K_{1}, K_{2}, \cdots, K_{h} \\
& \beta_{i}=M c_{i}>0 ; i=K_{1}, K_{2}, \cdots, K_{h} \text { and } \\
& \quad i \neq W_{1}, W_{2}, \cdots, W_{n_{r}} \\
& \beta_{i}=M c_{i}+\alpha_{i}>0 ; i=W_{1}, W_{2}, \cdots, W_{n_{r}}
\end{aligned}
$$

Now we let

$$
\begin{array}{r}
\boldsymbol{u}^{+}(N-j-1)=\left[\beta_{j m+1}, \beta_{j m+2}, \cdots, \beta_{j m+m}\right]^{T} ; \\
j=0,1, \cdots, N-1
\end{array}
$$

Then applying the positive control sequence $\left\{\boldsymbol{u}^{+}(0)\right.$, $\left.\boldsymbol{u}^{+}(1), \cdots, \boldsymbol{u}^{+}(N-1)\right\}$ to system $S_{r}$, we get $\boldsymbol{x}_{r}(N)=\mathbf{0}$ for any initial state $\boldsymbol{x}_{r}(0)$. Thus, from Definition 2, system $S_{r}$ is positive controllable.

Necessity : If we suppose that system $S_{r}$ is positive controllable, then by Theorem 2 and Lemma 3, we can select $n_{r}$ independent vectors $\left\{\boldsymbol{e}_{W_{1}}, \boldsymbol{e}_{W_{2}}, \cdots, \boldsymbol{e}_{W_{n}}\right\}$ out of $N m$ vectors $\left\{\boldsymbol{e}_{1}, \boldsymbol{e}_{2}, \cdots, \boldsymbol{e}_{N m}\right\}$ in (11). Now let

$$
\boldsymbol{x}^{*}=\boldsymbol{e}_{W_{1}}+\boldsymbol{e}_{W_{2}}+\cdots+\boldsymbol{e}_{W_{n_{r}}}
$$

Then $-x^{*} \in R^{n_{r}}$. Since system $S_{r}$ is positive controllable, there exist a finite positive integer $N$ and nonnegative scalars $\left\{d_{1}, d_{2}, \cdots, d_{N m}\right\}$ such that

$$
-x^{*}=d_{1} e_{1}+d_{2} e_{2}+\cdots+d_{N m} e_{N m}
$$

by Lemma 1. Adding (A.8) and (A.9) yields

$$
d_{1}^{*} \boldsymbol{e}_{1}+d_{2}^{*} \boldsymbol{e}_{2}+\cdots+d_{N m}^{*} \boldsymbol{e}_{N m}=0
$$

where

$$
\begin{gathered}
d_{i}^{*}=d_{i} \geqq 0 ; \\
\quad i=1,2, \cdots, N m \text { and } \\
i \neq W_{1}, W_{2}, \cdots, W_{n_{r}} \\
d_{i}^{*}=1+d_{i}>0 ; i=W_{1}, W_{2}, \cdots, W_{n_{r}}
\end{gathered}
$$

in (A.11) are zeros, then we obtain

$$
d_{W_{1}}^{*} e_{W_{1}}+d_{W_{2}}^{*} e_{W_{2}}+\cdots+d_{W_{r}}^{*} e_{W_{n_{r}}}=\mathbf{0}
$$

from (A.10). On the other hand, $n_{r}$ vectors $\left\{\boldsymbol{e}_{W_{1}}, \boldsymbol{e}_{W_{2}}\right.$, $\left.\cdots, \boldsymbol{e}_{W_{n}}\right\}$ are linearly independent.

Thus from(A.13), we obtain

$$
d_{i}^{*}=0 ; i=W_{1}, W_{2}, \cdots, W_{n r}
$$

This contradicts (A.12). Therefore, at least one of the $\left(N m-n_{r}\right)$ scalars $d_{i}^{*}$ 's in (A.11) is not zero. Now let $h$ be the number of positive $d_{i}^{* \prime} s$ in (A.10) and $\left\{\boldsymbol{f}_{1}\right.$, $\left.\boldsymbol{f}_{2}, \cdots, \boldsymbol{f}_{N m}\right\}$ be an appropreate rearrangement of $\left\{\boldsymbol{e}_{1}, \boldsymbol{e}_{2}\right.$, $\left.\cdots, \boldsymbol{e}_{N m}\right\}$.

Then, it follows from (A.10) (A.12) that

$$
g_{1} \boldsymbol{f}_{1}+g_{2} \boldsymbol{f}_{2}+\cdots+g_{h} \boldsymbol{f}_{h}=0
$$

where

$$
\begin{aligned}
& g_{i}>0 ; i=1,2, \cdots, h \\
& \operatorname{rank}\left[\boldsymbol{f}_{1}, \boldsymbol{f}_{2}, \cdots, \boldsymbol{f}_{h}\right]=n_{r}<h \leqq N m
\end{aligned}
$$

(A.15) $\sim($ A.17) are equivalent to (12) $\sim(14)$.

Therefore, the necessity is established. Q.E.D.

\section{《Appendix B》}

\section{Proof of Theorem 4}

Necessity: Since system $S_{q}$ is the subsystem of $S_{r}$, the necessity is clear by Lemma 2 .

Sufficiency: Suppose that conditions (1) and (2) hold. Then, by Lemma 4 , there exist a positive integer $N_{q}$ and nonnegative vector $\boldsymbol{\Omega}$ such that

$$
\left\langle\boldsymbol{A}_{q}, \boldsymbol{B}_{q}, N_{q}\right\rangle \boldsymbol{\Omega}=\mathbf{0} ; \boldsymbol{\Omega} \geqq \mathbf{0}
$$

where

$$
\begin{aligned}
& \boldsymbol{\Omega} \triangleq\left[\boldsymbol{\omega}_{1}^{T}, \boldsymbol{\omega}_{2}^{T}, \cdots, \boldsymbol{\omega}_{N_{q}}^{T}\right]^{T} ; \boldsymbol{\omega}_{1}>\mathbf{0} \\
& \boldsymbol{\omega}_{i} \in R^{m} ; i=1,2,3, \cdots, N_{q}
\end{aligned}
$$

Next, by a nonsingular transformation, we have the following equation from (41) and (42).

$$
\boldsymbol{A}_{r}=\left[\begin{array}{cc}
\boldsymbol{A}_{t} & \mathbf{0} \\
\mathbf{0} & \boldsymbol{A}_{q}
\end{array}\right], \boldsymbol{B}_{r}=\left[\begin{array}{c}
\boldsymbol{B}_{t} \\
\boldsymbol{B}_{q}
\end{array}\right]
$$

On the other hand, it has been proved in 7) that there exists a polynomial $\phi(z)$ with positive coefficients, such that

$$
\begin{aligned}
& \phi(z)=\delta_{L} z^{L}+\cdots+\delta_{1} z+\delta_{0} \\
& \delta_{i}>0 ; i=0,1,2, \cdots, L \\
& \phi\left(\boldsymbol{A}_{t}\right)=\mathbf{0}
\end{aligned}
$$

where $L$ may be designated arbitrarily as far as

$$
L \gg n_{t} \geqq 1
$$

Thus, from (B.4) (B.8), we obtain

$$
\phi\left(\boldsymbol{A}_{r}\right)=\sum_{i=0}^{L} \delta_{i} \boldsymbol{A}_{r}^{i}=\sum_{i=0}^{L} \delta_{i}\left[\begin{array}{cc}
\boldsymbol{A}_{t} & \mathbf{0} \\
\mathbf{0} & \boldsymbol{A}_{q}
\end{array}\right]^{i}
$$

If we assume that all of the $\left(N m-n_{r}\right)$ scalars $d_{i}^{*}$ 's 


$$
\begin{aligned}
& =\left[\begin{array}{cc}
\sum_{i=0}^{L} \delta_{i} \boldsymbol{A}_{t}^{i} & 0 \\
0 & \sum_{i=0}^{L} \delta_{i} \boldsymbol{A}_{q}^{i}
\end{array}\right] \\
& =\left[\begin{array}{cc}
\phi\left(\boldsymbol{A}_{t}\right) & 0 \\
\mathbf{0} & \phi\left(\boldsymbol{A}_{q}\right)
\end{array}\right]=\left[\begin{array}{cc}
\mathbf{0} & \mathbf{0} \\
\mathbf{0} & \phi\left(\boldsymbol{A}_{q}\right)
\end{array}\right]
\end{aligned}
$$

Therefore we get

$$
\begin{array}{r}
\phi\left(\boldsymbol{A}_{r}\right)\left\langle\boldsymbol{A}_{r}, \boldsymbol{B}_{r}, N_{q}\right\rangle \boldsymbol{\Omega} \\
=\left[\begin{array}{cc}
\mathbf{0} & \mathbf{0} \\
\mathbf{0} & \phi\left(\boldsymbol{A}_{q}\right)
\end{array}\right]\left[\begin{array}{l}
\left\langle\boldsymbol{A}_{t}, \boldsymbol{B}_{t}, N_{q}\right\rangle \\
\left\langle\boldsymbol{A}_{q}, \boldsymbol{B}_{q}, N_{q}\right\rangle
\end{array}\right] \boldsymbol{\Omega} \\
\quad=\left[\begin{array}{c}
\mathbf{0} \\
\phi\left(\boldsymbol{A}_{q}\right)\left\langle\boldsymbol{A}_{q}, \boldsymbol{B}_{q}, N_{q}\right\rangle \boldsymbol{\Omega}
\end{array}\right]=\mathbf{0}
\end{array}
$$

considering (B.1) and (B.2). Here, we can designate $L$ as $L \gg N_{q}$ and $L \gg n_{r}$, from (B.8). If we let

$$
\begin{aligned}
\omega_{i}^{*} & \triangleq \delta_{i-1} \omega_{1}+\delta_{i-2} \omega_{2}+\cdots+\delta_{i-N_{q}} \omega_{N_{q}} \in R^{m} ; \\
i & =1,2, \cdots, L+N_{q}
\end{aligned}
$$

where

$$
\delta_{i} \triangleq 0 ; i<0, i>L
$$

then (B.10) may be rewritten as;

$$
\left\langle\boldsymbol{A}_{r}, \boldsymbol{B}_{r}, L+N_{q}\right\rangle \boldsymbol{\Omega}^{*}=\mathbf{0} ; \boldsymbol{\Omega}^{*} \geqq 0
$$

where

$$
\begin{aligned}
& \Omega^{*} \triangleq\left[\omega_{1}^{* T}, \omega_{2}^{* T}, \cdots, \omega_{L+N_{q}}^{* T}\right]^{T}, \\
& \omega_{1}^{*} \triangleq \delta_{0} \omega_{1}>\mathbf{0}
\end{aligned}
$$

Hence, by Lemma 4, system $S_{r}$ is positive controllable.

Q.E. D.

\section{《Appendix C》}

\section{Proof of Theorem 5}

Necessity: Since system $S_{a}$ and $S_{b}$ are the subsystems of $S_{q}$, the necessity is clear.

Sufficiency: By a nonsingular transformation, we have

$$
\boldsymbol{A}_{q}=\left[\begin{array}{cc}
\boldsymbol{A}_{a} & 0 \\
\mathbf{0} & \boldsymbol{A}_{b}
\end{array}\right], \boldsymbol{B}_{q}=\left[\begin{array}{c}
\boldsymbol{B}_{a} \\
\boldsymbol{B}_{b}
\end{array}\right]
$$

from (39) and (40). Next, let

$$
\boldsymbol{B}_{q} \boldsymbol{E}_{m} \triangleq\left[\boldsymbol{x}_{a 0}^{T}, \boldsymbol{x}_{b 0}^{T}\right]^{T} \in R^{n_{q}}
$$

where $x_{a 0} \in R^{n_{a}}, x_{b 0} \in R^{n_{b}}$. Then, from Lemma 1, there exsist a positive integer $N_{a}$ and a nonnegative vector $\boldsymbol{\Omega}_{a}$, such that

$$
\begin{aligned}
& \left.\| \boldsymbol{A}_{a}, \boldsymbol{B}_{a}, N_{a}\right\rangle \boldsymbol{\Omega}_{a}=-x_{a 0} \in R^{n_{a}}, \\
& \mathbf{0} \leqq \boldsymbol{\Omega}_{a} \in R^{N_{a} m}
\end{aligned}
$$

since $S_{a}$ is positive controllable. Now, let

$$
\begin{aligned}
& \boldsymbol{\Omega}_{a}^{*} \triangleq\left[\boldsymbol{E}_{m}^{T}, \mathbf{0}\right]^{T}+\boldsymbol{\Omega}_{a} \in R^{N_{a} m} \\
& \boldsymbol{x}_{b}^{*} \triangleq\left\langle\boldsymbol{A}_{b}, \boldsymbol{B}_{b}, N_{a}\right\rangle \boldsymbol{\Omega}_{a}^{*} \in R^{n_{b}}
\end{aligned}
$$

Further, we consider a sufficiently large integer $N_{c}$ $\gg 1$. Then, from Lemma 1 , there exist a positive integer $N_{b}$ and a nonnegative vector $\boldsymbol{\Omega}_{b}$, such that

$$
\begin{aligned}
& \quad\left\langle\boldsymbol{A}_{b}, \boldsymbol{B}_{b}, N_{b}\right\rangle \boldsymbol{\Omega}_{b} \\
& \quad=-\left(\lambda_{b}\right)^{N_{c}} \boldsymbol{A}_{b}^{-N_{a}-N_{c}} x_{b}^{*} \in R^{N_{b}} \\
& \mathbf{0} \leqq \boldsymbol{\Omega}_{b} \in R^{N_{b} m}
\end{aligned}
$$

where $\lambda_{b}>0$ is any eigenvalue of $\boldsymbol{A}_{b}$, because $S_{b}$ is positive controllable. Next let

$$
\begin{aligned}
& N_{q} \triangleq N_{a}+N_{c}+N_{b} \\
& \boldsymbol{\Omega}_{q} \triangleq\left[\boldsymbol{\Omega}_{a}^{* T}, \mathbf{0}, \lambda_{b}^{-N_{c}} \boldsymbol{\Omega}_{b}^{T}\right]^{T} \in R^{N_{q} m} \\
& {\left[\boldsymbol{\omega}_{1}^{T}, \boldsymbol{\omega}_{2}^{T}, \cdots, \boldsymbol{\omega}_{N_{q}}^{T}\right]^{T} \triangleq \boldsymbol{\Omega}_{q}} \\
& \boldsymbol{\omega}_{i} \in R^{m} ; i=1,2, \cdots, N_{q}
\end{aligned}
$$

Then, from $($ C.1 $) \sim($ C.11), we obtain

$$
\begin{aligned}
& \left\langle\boldsymbol{A}_{q}, \boldsymbol{B}_{q}, N_{q}\right\rangle \boldsymbol{\Omega}_{\boldsymbol{q}} \\
& =\left[\begin{array}{c}
\left\langle\boldsymbol{A}_{a}, \boldsymbol{B}_{a}, N_{q}\right\rangle \\
\left\langle\boldsymbol{A}_{b}, \boldsymbol{B}_{b}, N_{q}\right\rangle
\end{array}\right]\left[\boldsymbol{\Omega}_{a}^{* T}, \mathbf{0}, \lambda_{b}^{-N_{c}} \boldsymbol{\Omega}_{b}^{T}\right]^{T} \\
& =\left[\boldsymbol{\varepsilon}_{a}^{T}, \mathbf{0}\right]^{T}
\end{aligned}
$$

and

$$
\begin{aligned}
& \boldsymbol{\Omega}_{\boldsymbol{q}} \geqq \mathbf{0} ; \boldsymbol{\omega}_{\mathbf{1}} \geqq \mathbf{0} \\
& \boldsymbol{\varepsilon}_{a} \triangleq\left(\lambda_{b}^{-1} \boldsymbol{A}_{a}\right)^{N_{c}} \boldsymbol{A}_{a}^{N_{a}}\left\langle\boldsymbol{A}_{a}, \boldsymbol{B}_{a}, N_{b}\right\rangle \boldsymbol{\Omega}_{b} \in R^{n_{a}}
\end{aligned}
$$

Here, all of the eigenvalues of the matrix $\lambda_{b}^{-1} \boldsymbol{A}_{a}$ are smaller than unity. Thus the norm of $\varepsilon_{a}$ is sufficiently small, because $N_{c}$ is sufficiently large. Hence, by Lemma 6 , system $S_{q}$ is positive controllable.

Q. E. D.

\section{《Appendix D》}

\section{Proof of Lemma 8}

Since system $S^{*}$ is the subsystem of $S_{G}^{+}$, the necessity is clear by Lemma 2

We will show the sufficiency by mathematical induction method.

When $G=1$, system $S_{1}^{+}$coinsides with system $S^{*}$. Therefore, if $S^{*}$ is positive controllable then $S_{1}^{+}$is positive controllable.

Next, we assume that for some positive integer $G \geqq$ 1 , the following two conditions hold:

(1) system $S^{*}$ is positive controllable.

(2) $S_{G}^{+}$is positive controllable.

Under these hypotheses, we will show that $S_{G+1}^{+}$is positive controllable by Lemma 6 , in the following 5 steps.

Step 1 : First, let

$$
\boldsymbol{x}_{G}^{*} \triangleq\left[\mathbf{0}, \boldsymbol{I}_{F G}\right] \boldsymbol{\Gamma}_{G+1} \boldsymbol{E}_{\boldsymbol{m}} \in R^{F G}
$$

Step 2 : Since system $S_{G}^{+}$is positive controllable by assumption (2), then by Lemma 1, there exist a positive integer $N_{G}$ and a nonnegative vector $\boldsymbol{\Omega}_{G}$, such that 
H. Y OShIDA, Y. T ANADA, T. T ANAKA and K. Y UNOKUCHI : Controllability with Positive Controls

$$
\begin{array}{ll}
\left\langle\boldsymbol{\Phi}_{G}, \boldsymbol{\Gamma}_{G} ; N_{G}\right\rangle \boldsymbol{\Omega}_{G}=-\boldsymbol{x}_{G}^{*} \in R^{F G} ; \boldsymbol{\Omega}_{G} \geqq 0 & \text { (D.2) } \\
\boldsymbol{\Omega}_{G} \in R^{N_{G} m} & \text { (D.3) }
\end{array}
$$

Step 3 : Let

$$
\begin{aligned}
& \boldsymbol{\Omega}_{G}^{*} \triangleq\left[\boldsymbol{E}_{m}^{T}, \mathbf{0}\right]^{T}+\boldsymbol{\Omega}_{G} \in R^{N_{G} m} \\
& \boldsymbol{x}^{o} \triangleq\left[\boldsymbol{I}_{\boldsymbol{F}}, \mathbf{0}\right]\left\langle\boldsymbol{\Phi}_{G+1}, \boldsymbol{\Gamma}_{G+1}, N_{G}\right\rangle \boldsymbol{\Omega}_{G}^{*} \in R^{\boldsymbol{F}}
\end{aligned}
$$

Step 4 : Since system $S^{*}$ is positive controllable by assumption (1), there exists a nonnegative vector $\boldsymbol{\Omega}_{0}$, such that

$$
B^{*} \boldsymbol{\Omega}_{o}=-x^{o} ; 0 \leqq \boldsymbol{\Omega}_{o} \in R^{m}
$$

\section{from Lemma 7.}

Step 5: Let $N$ be a positive integer such that $N \gg$ $N_{G}$. Further let

$$
\begin{aligned}
& \boldsymbol{\Omega}^{+} \triangleq\left[\boldsymbol{\Omega}_{G}^{* T}, \mathbf{0}, \eta \boldsymbol{\Omega}_{o}^{T}\right]^{T} \in R^{(N+1) m} \\
& \left\langle\boldsymbol{\Phi}_{G+1}, \boldsymbol{\Gamma}_{G+1}, N+1\right\rangle \boldsymbol{\Omega}^{+} \triangleq \varepsilon \in R^{F(G+1)} \\
& \eta \triangleq \lambda^{G-N}\left({ }_{N} \mathrm{C}_{G}\right)^{-1} \\
& { }_{N} \mathrm{C}_{G}=\frac{N !}{G !(N-G) !}
\end{aligned}
$$

To complete the proof by Lemma 8 , it is enough to show that

$$
\begin{aligned}
& \text { (i) } \boldsymbol{\Omega}^{+} \geqq \mathbf{0}, \boldsymbol{\omega}_{1}>\mathbf{0} \\
& \text { (ii) } \operatorname{rank}\left\langle\boldsymbol{\Phi}_{G+1}, \boldsymbol{\Gamma}_{G+1}, G+1\right\rangle=F(G+1) \\
& \text { (iii) the norm of } \boldsymbol{\varepsilon} \text { is sufficiently small. }
\end{aligned}
$$

Firstly, (i ) is derived from (D.2), (D.3), (D.5), (D.6), (D.8) and (D.9).

Secondly, we will show (ii) as follows.

From (53) and (61) we get

$$
\boldsymbol{\Phi}_{G+1}=\lambda \boldsymbol{I}_{F(G+1)}+\boldsymbol{N}
$$

where

$$
N \triangleq\left[\begin{array}{ccccc}
0 & \boldsymbol{I}_{F} & 0 & \cdots & 0 \\
0 & 0 & \boldsymbol{I}_{F} & \cdots & 0 \\
& \cdots & \cdots & \cdots & \\
0 & \cdots & 0 & 0 & \boldsymbol{I}_{F} \\
0 & \cdots & 0 & 0 & 0
\end{array}\right] \in R^{F(G+1) \times F(G+1)}
$$

Then we have from (61) and (D.13)

$$
\begin{aligned}
\operatorname{rank} & \left\langle\boldsymbol{\Phi}_{G+1}, \boldsymbol{\Gamma}_{G+1}, G+1\right\rangle \\
= & \operatorname{rank}\left[\boldsymbol{\Gamma}_{G+1}, \boldsymbol{\Phi}_{G+1}, \boldsymbol{\Gamma}_{G+1}, \cdots, \boldsymbol{\Phi}_{G+1} \boldsymbol{\Gamma}_{G+1}\right] \\
= & \operatorname{rank}\left[\boldsymbol{\Gamma}_{G+1},\left\{\lambda \boldsymbol{I}_{F(G+1)}+\boldsymbol{N}\right\} \boldsymbol{\Gamma}_{G+1}, \cdots,\right. \\
& \left.\left\{\lambda \boldsymbol{I}_{F(G+1)}+\boldsymbol{N}\right\}^{G} \boldsymbol{\Gamma}_{G+1}\right] \\
= & \operatorname{rank}\left[\boldsymbol{\Gamma}_{G+1}, \boldsymbol{N} \boldsymbol{\Gamma}_{G+1}, \cdots, \boldsymbol{N}^{G} \boldsymbol{\Gamma}_{G+1}\right] \\
= & \operatorname{rank}\left[\begin{array}{cccc}
\boldsymbol{B}_{G+1}^{+} & \boldsymbol{B}_{G}^{+} & \cdots & \boldsymbol{B}_{1}^{+} \\
\boldsymbol{B}_{G}^{+} & \boldsymbol{B}_{G-1}^{+} & \cdots & \mathbf{0} \\
\cdots & \cdots & \cdots & \cdots \\
\boldsymbol{B}_{2}^{+} & \boldsymbol{B}_{1}^{+} & \cdots & \mathbf{0} \\
\boldsymbol{B}_{1}^{+} & \mathbf{0} & \cdots & \mathbf{0}
\end{array}\right] \\
= & (G+1) \operatorname{rank}\left[\boldsymbol{B}_{1}^{+}\right]=(G+1) \operatorname{rank}\left[\boldsymbol{B}^{*}\right]
\end{aligned}
$$

On the other hand, system $S^{*}$ is positive controllable by assumption (1). Thus from (56) in Theorem 7 , rank $\left[\boldsymbol{B}^{*}\right]=F$. Therfore, we have

$$
\operatorname{rank}\left\langle\Phi_{G+1}, \Gamma_{G+1}, G+1\right\rangle=F(G+1)
$$

Lastly we will show (iii) as follows.

From (D.6) and (D.7), it follows taht

$$
\varepsilon=\left\langle\Phi_{G+1}, \Gamma_{G+1}, N_{G}\right\rangle \boldsymbol{\Omega}_{G}^{*}+\eta \Phi_{G+1}^{N} \Gamma_{G+1} \Omega_{o}
$$

Furtheremore, from (D.3), (D.1) and (D.2), we get

$$
\begin{aligned}
& {\left[0, \boldsymbol{I}_{F G}\right]\left\langle\boldsymbol{\Phi}_{G+1}, \boldsymbol{\Gamma}_{G+1}, N_{G}\right\rangle \boldsymbol{\Omega}_{G}^{*} } \\
& \quad=\left[\mathbf{0}, \boldsymbol{I}_{F G}\right]\left\langle\boldsymbol{\Phi}_{G+1}, \boldsymbol{\Gamma}_{G+1}, N_{G}\right\rangle \boldsymbol{\Omega}_{G}+\left[\mathbf{0}, \boldsymbol{I}_{F G}\right] \boldsymbol{\Gamma}_{G+1} \boldsymbol{E}_{m} \\
&=\left\langle\boldsymbol{\Phi}_{G}, \boldsymbol{\Gamma}_{G}, N_{G}\right\rangle \boldsymbol{\Omega}_{G}+\boldsymbol{x}_{G}^{*}=\mathbf{0}(\text { D. } 16)
\end{aligned}
$$

Thus from (D.4) and (D.16) we have

$$
\begin{aligned}
& \left\langle\boldsymbol{\Phi}_{G+1}, \boldsymbol{\Gamma}_{G+1}, N_{G}\right\rangle \boldsymbol{\Omega}_{G}^{*} \\
& =\left[\begin{array}{cc}
\boldsymbol{I}_{\boldsymbol{F}} & \mathbf{0} \\
\mathbf{0} & \boldsymbol{I}_{F G}
\end{array}\right]\left\langle\boldsymbol{\Phi}_{G+1}, \boldsymbol{\Gamma}_{G+1}, N_{G}\right\rangle \boldsymbol{\Omega}_{G}^{*} \\
& =\left[\begin{array}{cc}
\left.\left[\begin{array}{cc}
\boldsymbol{I}_{F} & 0
\end{array}\right] 《 \boldsymbol{\Phi}_{G+1}, \boldsymbol{\Gamma}_{G+1}, N_{G}\right\rangle \boldsymbol{\Omega}_{G}^{*} \\
{\left[\begin{array}{cc}
0 & \boldsymbol{I}_{F G}
\end{array}\right]\left\langle\boldsymbol{\Phi}_{G+1}, \Gamma_{G+1}, N_{G}\right\rangle \boldsymbol{\Omega}_{G}^{*}}
\end{array}\right] \\
& =\left[\left(\boldsymbol{x}^{o}\right)^{T}, \mathbf{0}\right]^{T}
\end{aligned}
$$

On the other hand, from (53), (61) and (D.8), we obtain

$$
\eta \boldsymbol{\Phi}_{G+1}^{N}=\left[\begin{array}{ccccc}
\boldsymbol{\Lambda}_{G} & \boldsymbol{\Lambda}_{G-1} & \cdots & \boldsymbol{\Lambda}_{1} & \boldsymbol{I}_{F} \\
\mathbf{0} & \boldsymbol{\Lambda}_{G} & \cdots & \boldsymbol{\Lambda}_{2} & \boldsymbol{\Lambda}_{1} \\
& \cdots & \cdots & \cdots & \\
\mathbf{0} & \mathbf{0} & \cdots & \boldsymbol{\Lambda}_{G} & \boldsymbol{\Lambda}_{G-1} \\
\mathbf{0} & \mathbf{0} & \cdots & \mathbf{0} & \boldsymbol{\Lambda}_{G}
\end{array}\right]
$$

where

$$
\boldsymbol{\Lambda}_{i} \triangleq \lambda^{i}\left({ }_{N} \mathrm{C}_{G}\right)^{-1}{ }_{N} \mathrm{C}_{G-i} \boldsymbol{I}_{F} \in R^{F \times F} ; i=1,2, \cdots, G
$$

If we let

$$
\begin{aligned}
& \eta \boldsymbol{\Phi}_{G+1}^{N} \boldsymbol{\Gamma}_{G+1} \boldsymbol{\Omega}_{o} \\
& \quad \triangleq\left[\tilde{\boldsymbol{x}}_{G+1}^{N}, \boldsymbol{\varepsilon}_{G}^{T}, \cdots, \varepsilon_{1}^{T}\right]^{T} \in R^{F(G+1)}
\end{aligned}
$$

then we have the following equations from (D.5), (D.18) and (61).

$$
\begin{aligned}
& \tilde{\boldsymbol{x}}_{G+1} \triangleq \boldsymbol{\varepsilon}_{G+1}-\boldsymbol{x}^{o} \in R^{F} \\
& \boldsymbol{\varepsilon}_{G+1} \triangleq\left(\boldsymbol{\Lambda}_{G} \boldsymbol{B}_{G+1}^{+}+\boldsymbol{\Lambda}_{G-1} \boldsymbol{B}_{G}^{+}+\cdots+\boldsymbol{\Lambda}_{1} \boldsymbol{B}_{2}^{+}\right) \boldsymbol{\Omega}_{o} \in R^{F}
\end{aligned}
$$

$$
\begin{array}{r}
\boldsymbol{\varepsilon}_{i} \triangleq\left(\boldsymbol{\Lambda}_{G} \boldsymbol{B}_{i}^{+}+\boldsymbol{\Lambda}_{G-1} \boldsymbol{B}_{i-1}^{+}+\cdots+\boldsymbol{\Lambda}_{G-i+1} \boldsymbol{B}_{1}^{+}\right) \boldsymbol{\Omega}_{o} \in R^{F} ; \\
i=1,2, \cdots, G \quad \text { (D } 23)
\end{array}
$$

Substituting (D.17), (D.20) and (D.21) into (D.15), it follows that

$$
\varepsilon=\left[\boldsymbol{\varepsilon}_{G+1}^{T}, \boldsymbol{\varepsilon}_{G}^{T} \cdots, \boldsymbol{\varepsilon}_{1}^{T}\right]^{T}
$$

Since $N$ is sufficiently large, all of the elements of $\boldsymbol{\Lambda}_{i} s(i=1,2, \cdots, G)$ in (D.19) are sufficiently small. Thus all of the norms of $\varepsilon_{i}$ 's $(i=1,2, \cdots, G+1)$ are sufficiently small by (D.22) and (D.23). 
Hence, the norm of $\varepsilon$ is sufficiently small.

Therefore, $S_{G+1}^{+}$is positive controllable by Lemma 6 .

Q.E.D.

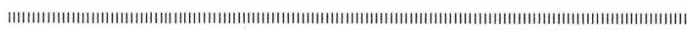

\section{[Authors' Profiles]}

Hiroshi Yoshida (Member)

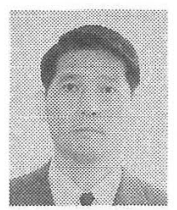

He received the Dr. Eng. degree in electronic engineering from Kyushu University, Fukuoka, Japan, in 1979. During 1966 and 1979, he was a research associate with the Department of Electronic Engineering, Kyushu University. From 1980 to 1987, he was an assistant professor with the Department of Electronic Engineering, Kagoshima University. Since 1988, he has been a professor at Kagoshima University. His main field of interest lies on digital control system theory.

\section{Yoshihiro Tanada (Member)}

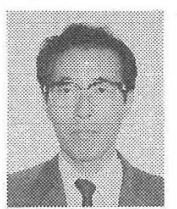

$\mathrm{He}$ received the B.E. degree from Okayama University in 1966 and the $\mathrm{Ph}$. D. degree from Kyoto University in 1979. In 1966, he was employed by Toyo Communication Equipment Co. From 1969 to 1988, he was with Okayama University and in 1988, was with Kagoshima University. Since 1991, he has been a professor of the Department of Information and Computer Science. His research interest includes orthogonal pseudonoise sequence and signal processing.

\section{Tetsuro Tanaka}

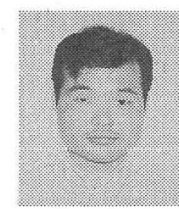

Tetsturo TANAKA was born in Kagoshima, Japan in 1961. He received the B.S., M.S., and Dr. Eng. degrees in electronics engineering from Kyushu University in Fukuoka, Japan, in 1983, 1985 , and 1992 respectively. He is presently an associate professor at Kagoshima University. His current interest is in the area of switching powerconverter, especially, noise reduction with random process. $\mathrm{He}$ is a member of IEICE.

\section{Kazutomo YUNOKUCHI}

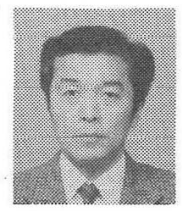

In 1987 he stayed at Institute of Medical engineering, University of Tokyo. $\mathrm{He}$ woked at Francis Bitter Magnet Laboratory in M.I.T. as a visiting researcher from 1989 to 1991 . He received Dr. Engineering degree from University of Tokyo in 1993. He is an associate professor of Department of Electronic Engineering, Kagoshima University. His research interest concentrates on the control system of human body. He is a member of the Japan society of medical engineering.

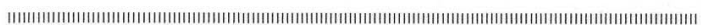

